\title{
Ion Acoustic Waves in Weakly Relativistic Plasma-Separation of Relativistic and Electron-Temperature Effects
}

\author{
Yair Zarmi \\ Jacob Blaustein Institutes for Desert Research, Ben-Gurion University of the Negev, Midreshet Ben-Gurion, 8499000, Israel.
}

How to cite this paper: Yair Zarmi. (2021) Ion Acoustic Waves in Weakly Relativistic Plasma-Separation of Relativistic and Electron-Temperature Effects. Journal of Applied Mathematics and Computation, 5(1), 32-47.

DOI: 10.26855/jamc.2021.03.005

Received: January 26, 2021

Accepted: February 25, 2021

Published: March 12, 2021

*Corresponding author: Yair Zarmi, Jacob Blaustein Institutes for Desert Research, Ben-Gurion University of the Negev, Midreshet Ben-Gurion, 8499000, Israel.

Email: zarmi@bgu.ac.il

\begin{abstract}
The choice of the expansion parameter employed in the analysis of the equations of weakly relativistic plasma affects the physical significance of the results. Traditionally, the small parameter employed in the non-relativistic and weak relativistic limits has been the order of magnitude of $\left(v / v_{0}\right)$, where $v$ is the ion velocity, and $v_{0}$ is proportional to the average electron velocity. However, in the weak relativistic case, the order of magnitude of $(\mathrm{v} / \mathrm{c})$, where $c$ is the speed of light is the more natural choice. The resulting KdV equation with perturbations through second order is analyzed through a Normal Form expansion. The analysis exposes physical effects in corrections beyond lowest order, which hitherto have not be identified: (1) Effect of localized soliton interaction region; (2) Long-range interactions among solitons; (3) Dispersive waves generated by soliton interactions. In addition, the analysis provides information regarding: (1) Clear distinction between non-relativistic and weak relativistic effects; (2) Clear separation between relativistic and electron-temperature effects; (3) Variation of the effective small parameter used in the series expansion of the solution as the average electron kinetic energy is increased. These qualitative features do not depend on the details of the electron-gas thermodynamic distribution.
\end{abstract}

\section{Keywords}

Ion Acoustic Waves, Weak Relativistic Effects, Normal Form Analysis, Average Electron Energy Effects, Soliton Interactions

\section{Introduction}

\subsection{Ion acoustic wave equations}

The ion acoustic wave equations describe the dynamics of collision-less plasma made of cold ions and hot electrons in $(1+1)$ dimensions. For decades, this system was analyzed in the non-relativistic limit through a perturbation expansion, yielding in lowest order the KdV equation and its soliton solutions. In recent years, works that account for small relativistic effects have been published. In some works, a lowest order analysis led to the KdV [1-15] or the Nonlinear Schrödinger equation [16-17]. In others, the analysis went as far as generating the KdV equation with a first-order perturbation [18-20].

The equations are:

The continuity equation,

$$
n_{t}+(n v)_{x}=0
$$


the momentum-conservation equation, which contains the relativistic effects,

$$
m\left\{(\gamma v)_{t}+v(\gamma v)_{x}\right\}+e \varphi_{x}=0,
$$

and the Poisson equation for the electrostatic potential,

$$
\varphi_{x x}=-4 \pi e\left\{n-n_{e}\right\} \quad, \quad\left(n_{e}=n_{0} \psi\left(e \varphi /\left(k_{B} T_{e}\right)\right)\right) .
$$

In Eqs. (1-3), $n, v, m$, and are the ion density, velocity and mass, respectively, $n_{e}$ is the distribution of the electrons, $n_{0}$ is the particle-number density in the unperturbed state of the plasma, $\varphi$ is the electric potential $e$ is the electron charge, $k_{B}$ is the Boltzmann constant and $T_{e}$ is the electron gas temperature. Finally, $\gamma$ in Eq. (2) is the Lorentz factor:

$$
\gamma=1 / \sqrt{1-(v / c)^{2}}
$$

where $c$ is the speed of light. In this paper, weakly relativistic effects $((v / c) \ll 1)$ will be studied.

In Eq. (3), $\psi\left(e \varphi /\left(k_{B} T_{e}\right)\right)$ represents the thermodynamics of the electron gas. $\psi$ may correspond to a Maxwell-Boltzmann- or a Fermi-Dirac-distribution, or other distributions of relevance in various physical situations. At infinite distances, the plasma is in its unperturbed state, $v \rightarrow 0, n_{e} \rightarrow n_{0}$ and $\varphi \rightarrow 0$. Hence, Eq. (3), requires that $\psi(0)=1$.

\subsection{Choice of velocity scale}

Finding small-amplitude soliton solutions and higher-order corrections to the solution requires the identification of a small parameter. To this end, one needs to identify a velocity scale, $v_{0}$, such that $\left(v / v_{0}\right) \ll 1$. The order of magnitude of this ratio then serves as the expansion parameter in a perturbative analysis.

\subsubsection{Traditional choice}

Past analyzes of the weak relativistic case [1-20], have adopted the expansion parameter that was used in the analysis of the non-relativistic limit $\left(\left(v / v_{0}\right) \rightarrow 0, \gamma=1\right.$ in Eq. (2)). This choice for $v_{0}$ was based on the assumption that the electrons obey a Boltzmann distribution, has been:

$$
v_{0}=\sqrt{\left(k_{B} T_{e} / m\right)}=v_{e} \sqrt{\left(\left(m_{e} / 2\right) / m\right)}=\alpha c \quad, \quad\left(\alpha=\sqrt{\left(k_{B} T_{e} / m c^{2}\right)}\right) .
$$

Here, $v_{e}$ is the average electron velocity and $m_{e}$ is the electron mass. $\alpha$ measures the smallness of the electron kinetic energy relative to the rest-mass energy of the ions. One now writes

$$
v / v_{0}=\varepsilon U,|\varepsilon|=1, U=O(1) .
$$

The Lorentz factor is re-written as:

$$
\gamma=1 / \sqrt{1-\left(v_{0} / c\right)^{2}\left(v / v_{0}\right)^{2}}=1 / \sqrt{1-\alpha^{2} \varepsilon^{2} U^{2}} .
$$

The choice of Eqs. (5) and (6) suffers from several disadvantages, discussed in Section 1.3.

\subsubsection{Natural velocity scale for weak relativistic effects}

Eqs. (5) and (6) do not constitute natural scaling for the study of weak relativistic effects. Wishing to account for the latter, calls for scaling $v$ relative to $c$ :

$$
v / c=\mu U, \quad|\mu| \ll 1 \quad, \quad U=O(1) .
$$

The Lorentz factor of Eq. (4) is re-written as:

$$
\gamma=1 / \sqrt{1-\rho \mu^{2} U^{2}},
$$

The fictitious constant $\rho(=1)$ in Eq. (9) has been introduced so as to identify relativistic effects.

\subsection{Advantages of scaling of Eq. (8)}

The scaling choice of Eqs. (5) and (6), adopted in the analysis of Eqs. (1-3) in the case of a weakly relativistic plasma $((\gamma-1) \ll 1)$ [1-20], may lead to confusion on several counts. For example, in a Normal Form analysis of Eq. (1-3) (see Section 4), this scaling choice yields for $v$ : 


$$
v \simeq \varepsilon c \alpha\left\{u_{0}+\varepsilon\left(\frac{1}{12}\left(8 u_{0}^{2}+14 u_{0, X} \partial_{X}^{-1} u_{0}+3 u_{0, X X}\right)+\frac{3}{8} \alpha\left(2 u_{0}^{2}+u_{0, X X}\right)\right)\right\}+O\left(\varepsilon^{2}\right) .
$$

$u_{0}=O(1)$ is the zero-order approximation to the solution.

Examining Eq. (10) one infers that $\varepsilon$ of Eq. (6) need not reflect the smallness of $(v / c)$, because, by Eq. (5), $v_{0} \ll v_{e}$. In fact, $v$ is doubly small relative to the speed of light, as (usually, $\alpha \ll 1$ ):

$$
v=O(\varepsilon \alpha) c \text {. }
$$

Furthermore, both $\alpha$ and $\varepsilon$ appear in the Lorentz factor in Eq. (7). As a result, there is no clear separation between non-relativistic, weak relativistic and electron temperature effects.

As a result, it is not obvious which part of the first-order correction in Eq. (10) corresponds to weak relativistic effects and which part- to electron temperature effects.

Finally, owing to the smallness of $\alpha$, what may be interpreted as the relativistic effect (the term linear in $\alpha$ in the first-order correction) is so small that the first-order approximation remains very close to the result of the non-relativistic limit.

Such confusion afflicts another aspect of an analysis that uses the scaling of Eqs. (5) and (6). The parameter that defines the smallness of $v$ in Eqs. (1-3) ( $\varepsilon$ in Eq. (6), and $\mu$ in Eq. (8)) is, usually, not the parameter employed in the expansion of the solution. The two are different. The analysis based on the traditional scaling (Eq. (6)) leads to the perturbed KdV equation:

$$
U_{T}=6 U U_{X}+U_{X X X}+\varepsilon F_{1}[U]+\varepsilon^{2} F_{2}[U]+\cdots
$$

( $T$ and $X$ are transformed dimensionless time and position coordinates.) All $F_{n}(n \geq 1)$ in Eq. (12) turn out to be differential polynomials in $U$. The solution for $U$ is written as a power series in $\varepsilon$ :

$$
U=u_{0}+\varepsilon u_{1}+\varepsilon^{2} u_{2}+\cdots
$$

Although $\varepsilon$ is the formal expansion parameterin Eqs. (12) and (13), when KdV solitons are considered, the effective parameter used in the expansion of the solution turns out to be

$$
\tilde{\varepsilon}=\varepsilon q^{2} \quad, \quad|\tilde{\varepsilon}| \ll 1 .
$$

Here $q$ is a soliton wave number. (In a multi-soliton solution, $q$ ought to be the largest wave number, that of the leading soliton.) In fact, $\tilde{\varepsilon}$ already appears in the single-soliton solution:

$$
u_{0}=\frac{2 q^{2}}{\cosh [q(X+V T)]},\left(V=4 q^{2}\left(1+\left(4 \varepsilon q^{2}\right) a_{1}+\left(4 \varepsilon q^{2}\right)^{2} a_{2}+\cdots\right)\right)
$$

$a_{i}$ are determined by the perturbation.

Furthermore, as $F_{n}(n \geq 1)$ in Eq. (12) are differential polynomials in $U$, one finds that when a single-soliton solution is considered then

$$
F_{n}=q^{5}\left(q^{2}\right)^{n} O(1) \quad, \quad u_{n}=q^{2}\left(q^{2}\right)^{n} O(1) \quad n \geq 0 .
$$

Again, the expansion parameter is $\tilde{\varepsilon}$ of Eq. (14), whereas the smallness of ion velocity, $v$, is measured by $\varepsilon$. As $\varepsilon$ is measured relative to $v_{0}$ of Eqs. (5) and (6), it cannot separate the effect on $\tilde{\varepsilon}$ of the average electron kinetic energy from small relativistic effects. Both effects are hidden in $\tilde{\varepsilon}$.

In the analysis presented in the following Sections, where the scaling of Eq. (8) is employed, these difficulties are avoided, allowing for clear separation between relativistic effects, represented by $\mu=O(v / c)$, and the effect of the average electron velocity represented by $\alpha$. In particular, in the case of KdV solitons, the dependence of the effective expansion parameter in a Normal Form analysis of Eqs. (1-3) on the magnitude of $\alpha$ (see the detailed discussion in Section 9):

$$
\tilde{\mu}=\left\{\begin{array}{ll}
\left(\mu q^{2} / \alpha\right) & \alpha \ll 1 \\
\mu q^{2} & \alpha=O(1)
\end{array} .\right.
$$

Finally, a technical comment. In Eq. (10) and later on, the operator $\partial_{X}{ }^{-1}$ is defined as 


$$
\partial_{X}^{-1} f(X)=\frac{1}{2}\left(\int_{-\infty}^{X} f(X) d \quad X \int_{X}^{\infty} f(X) d X\right.
$$

\subsection{Electron distribution}

The analysis reveals that the electrostatic potential, $\varphi$, is of $O(\mu)$. As a result, in the expansion in powers of $\mu$, the resulting equations depend on $\psi\left(e \varphi / k_{B} T_{e}\right)$ only on $\psi(0)=1$ (see discussion following Eq. (4)), and $\psi^{(\mathrm{n})}(0)$. Thus, different choices of $\psi$ lead to different numerical coefficients in the perturbative expansion, but do not change the qualitative features of the results. Hence, for the sake of simplicity, results will be presented for a Maxwell-Boltzmann distribution, $\psi\left(e \varphi / k_{\mathrm{B}} T_{e}\right)=e^{e \varphi / k_{\mathrm{B}} T_{e}}$, for which all $\psi^{(\mathrm{n})}(0)=1$. Examples of how the results are modified for a general $\psi$ will be provided.

\subsection{Results presented in this paper}

Adopting the scaling of Eq. (8), a formal expansion in powers of $\mu$ generates an approximation for $v$ through a Normal Form procedure (see Section 4). The analysis, carried out through $O\left(\mu^{2}\right)$, provides information about the following aspects of the solution:

1) Weak relativistic effects;

2) Long-range interactions among solitons (Section 7);

3) Effects of soliton interaction region (Section 8);

4) Generation of dispersive waves by non-relativistic and relativistic terms as a consequence of soliton interactions (Section 8.2);

5) Effect of the magnitude of the average electron kinetic energy, $\left(k_{B} \cdot T_{e}\right)$ (Section 9).

\subsection{Generation of dispersive waves}

From second-order and onwards, the perturbation contains driving terms, which generate dispersive waves as corrections to the solution. These are discussed in Section 8.2. The effect of these new driving terms on the solution cannot be written in closed form as differential polynomials in the zero-order approximation (polynomials in the solution and its spatial derivatives). They have been called obstacles to asymptotic integrability [21-33]. The previous studies of weak relativistic effects [1-20] have missed these effects as they did not perform a second order analysis.

\subsection{Two small parameters vs. one small parameter}

The analysis of the solution of Eqs. (1-3) is controlled by two small parameters: $\mu$ (Eq. (8)), which represents the magnitude of ( $v / \mathrm{c}$ ) and $\alpha$ (Eq. (5)) (for $T_{e}=10^{6} \mathrm{~K}$ and $m$ - the proton mass, $\alpha \approx 10^{-7}$ ). As these parameters are unrelated, one might presume that an expansion in both is required. However, instead of the KdV equation, which has soliton solutions, a Normal Form expansion in the two parameters generates an uninteresting linear equation already in the lowest order:

$$
U_{t}+A U_{x x x}=0
$$

The value of the constant $A$ depends on the ample freedom inherent in the expansion procedure.

The details of this analysis are not included here. However, it carries an important message: To obtain the perturbed $\mathrm{KdV}$ equation one ought to restrict oneself to a single-parameter expansion. This has been done in the literature with the scaling of Eqs. (5) and (6) [1-20]. It will be also adopted here, but with the scaling of Eq. (8).

\section{Variable transformations}

\subsection{Variables}

The first stage is the reduction of the system of Eqs. (1-3) into one equation for $U$ of Eq. (8), to which a perturbation expansion is applied. Using Eq. (3), the ion density, $n$, is written as:

$$
n=n_{0} \psi\left(e \varphi / k_{B} T_{e}\right)-\frac{1}{4 \pi e} \varphi_{x x} .
$$

Using the scaling choice of Eq. (8), one writes $v$ and $\varphi$ of Eqs. (1-3) as: 


$$
\begin{gathered}
v(t, x)=\mu c U(T, X), \quad \varphi(t, x)=F(T, X) \frac{m c^{2}}{e} \\
\tau=\sqrt{4 \pi \frac{e^{2} n_{i j}}{k_{B} T_{c}} c t}, \quad \xi=\sqrt{4 \pi \frac{e^{2} n_{i j}}{k_{B} T_{e}} x}, \quad T=b \mu^{3 / 2} \tau, \quad X=d \mu^{1 / 2}\left(\xi-v_{g} \tau\right)
\end{gathered} .
$$

Substituting Eqs. (20) and (21) in Eqs. (1)-(3), and solving for $F(T, X)$ from Eq. (2), yields an equation for $U(T, X)$. The resulting equation is expanded in powers of $\mu$. In the analysis presented here the expansion has been carried out through $O\left(\mu^{2}\right)$. The constants, $v_{g}$ (velocity of the moving frame), $b$ and $d$ are chosen so as to generate for $U$ a perturbed KdV equation:

$$
U_{T}=6 U U_{X}+U_{X X X}+O(\mu) .
$$

The required values for $b$ and $d$ are found to be:

$$
\begin{array}{ll}
b=1 /(6 \sqrt{3 \alpha})\left(\left(3 \psi^{\prime}(0)^{2}-\psi(0) \psi^{\prime \prime}(0)\right)^{3 / 2} /\left(2 \psi^{\prime}(0)^{9 / 4}\right)\right) & \begin{array}{l}
\text { Maxwell-Boltzmann } \\
=
\end{array} /(6 \sqrt{3 \alpha}) \\
d=-1 / \sqrt{3 \alpha}\left(\psi^{\prime}(0)^{2}\left(3 \psi^{\prime}(0)^{2}-\psi(0) \psi^{\prime \prime}(0)\right)^{1 / 2} /\left(\sqrt{2} \psi^{\prime}(0)^{9 / 4}\right)\right) & \begin{array}{c}
\text { Maxwell-Boltzmann } \\
=
\end{array}-1 / \sqrt{3 \alpha}
\end{array} .
$$

In Eq. (23), the first values of $b$ and $d$ are obtained for a general distribution function, $\psi$ (see Eq. (3)), and the second values are obtained when $\psi$ is a Maxwell-Boltzmann distribution.

\subsection{Moving frame velocity}

A priory, one might expect the velocity of the moving frame, $v_{g}$, to depend on $\mu$ :

$$
v_{g}=v_{g}^{(0)}+\sum_{m \geq 1} \mu^{m} v_{g}^{(m)} \text {. }
$$

For a single soliton, one finds that $v_{g}^{(m)}, m \geq 1$,depend on the soliton wave number. Hence, in the case of a multi-soliton solution, away from the soliton interaction region, the correction along each soliton has to be different, as each soliton has a different wave number. Abandoning the power expansion, Eq. (24), one finds that $v_{g}$ depends on the average electron velocity (see Eq. (5)):

$$
v_{g}=\alpha / \sqrt{\psi^{\prime}(0)} \quad \underset{\text { Maxwell-Boltzmann }}{=} \alpha .
$$

\subsection{Electrostatic potential}

Assuming a vanishing boundary condition at $\pm \infty$, one solves Eq. (2) for $F(T, X)$, (see Eq. (21)):

$$
\begin{aligned}
& F(T, X)=1-\frac{1}{\sqrt{1-\mu^{2} U^{2}}}\left(1-\alpha \mu\left(1 / \psi^{\prime}(0)\right)^{1 / 2} U\right) \\
& +\frac{1}{12} \frac{\left(3 \psi^{\prime}(0)^{2}-\psi^{\prime \prime}(0)\right)}{\psi^{\prime}(0)^{2}} \mu^{2} \partial_{T} \partial_{X}^{-1}\left(\frac{U}{\sqrt{1-\mu^{2} U^{2}}}\right) \\
& \underset{\text { Maxwell-Boltzmann }}{=} 1-\frac{1}{\sqrt{1-\mu^{2} U^{2}}}(1-\alpha \mu U)+\frac{1}{6} \mu^{2} \partial_{T} \partial_{X}^{-1}\left(\frac{U}{\sqrt{1-\mu^{2} U^{2}}}\right) \text {. } \\
& \underset{\text { Maxwell-Boltzmann }}{=} \alpha \mu U+\frac{1}{6} \mu^{2}\left(\partial_{X}^{-1} U_{T}-3 U^{2}\right)+O\left(\mu^{3}\right)
\end{aligned}
$$

The effect of the average electron kinetic appears explicitly in the first-order term.

\section{Perturbed KdV equation}

For a Maxwell-Boltzmann distribution, the preceding steps yield the following perturbed $\mathrm{KdV}$ equation for $U(T, X)$ : 


$$
\begin{aligned}
U_{T}= & 6 U U_{X}+U_{X X X} \\
& -\mu\left\{\frac{1}{12 \alpha}\left(\partial_{X}^{-1} U_{T T}-36 U^{2} U_{X}+48 U_{X} U_{X X}+24 U U_{X X X}-4 U_{T X X}\right)+\rho \alpha \frac{9}{2} U^{2} U_{X}\right\} . \\
& -\mu^{2}\left\{\begin{array}{l}
\left.\frac{1}{36 \alpha^{2}}\left(\begin{array}{l}
3 U \partial_{X}^{-1} U_{T T}-36 U U_{X} \partial_{X}^{-1} U_{T}+108 U^{3} U_{X}-36\left(U_{X}\right)^{3} \\
-144 U U_{X} U_{X X}-36 U^{2} U_{X X X}+6 U_{T} \partial_{X}^{-1} U_{T}-36 U^{2} U_{T} \\
+6 U_{X X} U_{T}+18 U_{X} U_{T X}+12 U U_{T X X}-U_{T T X}
\end{array}\right)\right\}+O\left(\mu^{3}\right) \\
-\rho \frac{3}{2}\left(3 U^{3} U_{X}+2\left(U_{x}\right)^{3}+6 U U_{X} U_{X X}+U^{2} U_{X X X}-U^{2} U_{T}\right)
\end{array}\right\}
\end{aligned}
$$

For a general distribution, $\psi$, (see Eq. (3)), the numerical coefficients in Eq. (27) are modified. For example, in $O(\mu)$ in Eq. (27), the $U^{2} U_{X}$ and the $U U_{X X X}$ terms are modified as follows:

$$
\begin{gathered}
\left(-\frac{3}{\alpha}+\rho \alpha \frac{9}{2} U^{2} U_{X}\right) U^{2} U_{X} \rightarrow \\
\left(\frac{3}{\alpha} \frac{3 \psi^{\prime}(0)^{3}-6 \psi^{\prime}(0) \psi^{\prime \prime}(0)+\psi^{\prime \prime \prime}(0)}{\sqrt{\psi^{\prime}(0)}\left(3 \psi^{\prime}(0)^{2}-\psi^{\prime \prime}(0)\right)}+\rho \alpha \frac{9}{2} \frac{2 \sqrt{\psi^{\prime}(0)^{3}}}{3 \psi^{\prime}(0)^{2}-\psi(0) \psi^{\prime \prime}(0)}\right) U^{2} U_{X} \\
\frac{2}{\alpha} U U_{X X X} \rightarrow \frac{2}{\alpha} \sqrt{\psi^{\prime}(0)} U U_{X X X} .
\end{gathered}
$$

For the sake of simplicity, the results will be presented for the case of a Maxwell-Boltzmann distribution. Finally, note that the relativistic effects (multiplied by $\rho(=1)$ ) and the effect (through $\alpha$ ) of the average electron kinetic energy appear explicitly in Eq. (27). The qualitative aspects of the results presented here do not depend on the details of $\psi$.

\section{Normal Form expansion [21-29]}

One now expands $U(T, X)$ in powers of $\mu$ :

$$
U=U^{(0)}+\mu U^{(1)}+\mu^{2} U^{(2)}+O\left(\mu^{3}\right) .
$$

The analysis of Eq. (27) will be carried out through $O\left(\mu^{2}\right)$ because obstacles to asymptotic integrability emerge in the multi-soliton case only from $O\left(\mu^{2}\right)$ and onwards [21-33].

Resonant terms appearing in the dynamical equations generate secular terms in $U^{(n)}$ of Eq. (28) for $n \geq 1$. Secular terms limit the validity of the approximate solution to $T=O(1)$. The Normal Form analysis allows for extension of the validity in the case of soliton solutions to $T=O(1 / \tilde{\mu})$ (see Eq. (17)). To this end, one removes the resonant terms from the dynamical equations of $U^{(n)}$ and transfers them to the equation that governs the evolution of $U^{(0)}$, the Normal Form:

$$
U_{T}^{(0)}=S_{2}\left[U^{(0)}\right]+\mu a_{1} S_{3}\left[U^{(0)}\right]+\mu^{2} a_{2} S_{4}\left[U^{(0)}\right]+O\left(\mu^{3}\right) .
$$

The coefficients, $a_{1}$ and $a_{2}$ in Eq. (29) are determined by the structure of the perturbation. $S_{n}\left[U^{(0)}\right]$ are the resonant terms, also called symmetries of the KdV equation [23, 25, 26,28, 29]. Of the infinite hierarchy of symmetries, the following are used in the analysis through $O\left(\mu^{2}\right)$ :

$$
\begin{aligned}
& S_{1}=U_{X}^{(0)} \\
& S_{2}=6 U^{(0)} U_{X}^{(0)}+U_{X X X}^{(0)} \\
& S_{3}=30\left(U^{(0)}\right)^{2} U_{X}^{(0)}+10 U U_{X X X}^{(0)}+20 U_{X}^{(0)} U_{X X}^{(0)}+U_{X X X X X}^{(0)} \\
& S_{4}=140\left(U^{(0)}\right)^{3} U_{X}^{(0)}+70\left(U^{(0)}\right)^{2} U_{X X X}^{(0)}+280 U^{(0)} U_{X}^{(0)} U_{X X}^{(0)}+14 U^{(0)} U_{X X X X X}^{(0)}+ \\
& 70\left(U_{X}^{(0)}\right)^{3}+42 U_{X}^{(0)} U_{X X X X}^{(0)}+70 U_{X X}^{(0)} U_{X X X}^{(0)}+U_{X X X X X X X}^{(0)}
\end{aligned}
$$


Note that the first term in the Normal Form, Eq. (29), is the usual KdV term.

The Normal Form, Eq. (29), is integrable in the Inverse-Scattering sense. Its solutions are KdV solitons [23, 25, 26, 28, 29]. The higher-order terms merely modify individual soliton velocities. For example, the single-soliton solution of Eq. (29), is the usual KdV soliton:

$$
U_{\text {Single }}^{(0)}(T, X)=\frac{2 q^{2}}{\cosh [q(X+V T+\delta)]^{2}} .
$$

Here $q$ is the soliton wave number, and $\delta$ - a constant phase shift. In the unperturbed KdV equation, the soliton velocity, $V=4 q^{2}$. It is updatedby Eq. (29) into:

$$
V=4 q^{2}\left(1+\left(4 q^{2} \mu\right) a_{1}+\left(4 q^{2} \mu\right)^{2} a_{2}+\ldots\right) .
$$

A multi-soliton solution of Eq. (29) is also the usual KdV multi-soliton solution. Away from the soliton interaction region, where the solution breaks up into a sum of well-separated single solitons, the velocity of each soliton is updated by Eq. (32), with $q$ being its wave number.

\section{Structure of higher-order corrections to solution}

\subsection{First-order approximation}

As the perturbation terms in Eq. (27) are all polynomials in $U$ and its spatial derivatives (differential polynomials), it has been customary to assume that the higher-order terms, $U^{(n)}, n \geq 1$, in Eq. (28) are differential polynomials in $U^{(0)}$. This assumption works in all orders of the expansion when $U^{(0)}$ is a single soliton solution of Eq. (29). For general solutions, the hypothesis works in $O(\mu)$. The most general expression for $U^{(1)}$ is:

$$
U^{(1)}=c_{1} U_{X X}^{(0)}+c_{2} U_{X}^{(0)} q^{(1)}+c_{3} U^{(0)}\left(q^{(1)}\right)^{2}+c_{4}\left(U^{(0)}\right)^{2}+c_{5}\left(q^{(1)}\right)^{4}+c_{6} q^{(1)} q^{(3)}+c_{7} q^{(4)} .
$$

\subsection{Second-order approximation}

The differential polynomial hypothesis fails in $O\left(\mu^{2}\right)$ for multi-soliton solutions. The equation for $U^{(2)}$ contains a driving term, the contribution of which to $U^{(2)}$ cannot be described by the most general differential polynomial in $U^{(0)}$ consistent with the expansion procedure. Such a driving term has been called an obstacle to asymptotic integrability [21-33]. It generates dispersive waves that run away from the soliton trajectories (See Section 8.2.). One, therefore, writes $U^{(2)}$ as the most general differential polynomial plus a term, $W(T, X)$, to account for the effect of the obstacle:

$$
\begin{aligned}
& U^{(2)}(T, X)= \\
& d_{1} U_{X X X X}^{(0)}+d_{2} U_{X X X}^{(0)} q^{(1)}+d_{3} U_{X X}^{(0)}\left(q^{(1)}\right)^{2}+d_{4} U_{X}^{(0)}\left(q^{(1)}\right)^{3}+d_{5} U_{X}^{(0)} q^{(3)} \\
& +d_{6} U^{(0)}\left(q^{(1)}\right)^{4}+d_{7} U^{(0)} q^{(1)} q^{(3)}+d_{8} U^{(0)} q^{(4)}+d_{9} U^{(0)} U_{X X}^{(0)} \\
& +d_{10}\left(U_{X}^{(0)}\right)^{2}+d_{11} U^{(0)} U_{X}^{(0)} q^{(1)}+d_{12}\left(U^{(0)}\right)^{2}\left(q^{(1)}\right)^{2}+d_{13}\left(U^{(0)}\right)^{3} \\
& +d_{14} q^{(6,1)}+d_{15} q^{(6,2)}+d_{16} q^{(6,3)}+d_{17} q^{(1)} q^{(5,1)}+d_{18} q^{(1)} q^{(5,2)} \\
& +d_{19} q^{(1)} q^{(5,3)}+d_{20}\left(q^{(1)}\right)^{2} q^{(4)}+d_{21}\left(q^{(1)}\right)^{3} q^{(3)}+d_{22}\left(q^{(3)}\right)^{2}+d_{23}\left(q^{(1)}\right)^{6} \\
& +W(T, X)
\end{aligned}
$$

The justification for Eqs. (33) and (34) has been discussed extensively [25, 26, 28, 29, 34]. In Eqs. (33) and (34),

$$
\begin{aligned}
& q^{(1)}=\partial_{X}^{-1}\left(U^{(0)}\right), \quad q^{(3)}=\partial_{X}^{-1}\left(\left(U^{(0)}\right)^{2}\right), \quad q^{(4)}=\partial_{X}^{-1}\left(\left(U^{(0)}\right)^{2} q^{(1)}\right) \\
& q^{(5,1)}=\partial_{X}^{-1}\left(\left(U^{(0)}\right)^{3}\right), \quad q^{(5,2)}=\partial_{X}^{-1}\left(\left(U^{(0)}\right)^{2}\left(q^{(1)}\right)^{2}\right), \quad q^{(5,3)}=\partial_{X}^{-1}\left(\left(U_{X}^{(0)}\right)^{2}\right) \\
& q^{(6,1)}=\partial_{X}^{-1}\left(\left(U^{(0)}\right)^{3} q^{(1)}\right), \quad q^{(6,2)}=\partial_{X}^{-1}\left(\left(U^{(0)}\right)^{2}\left(q^{(1)}\right)^{3}\right), \quad q^{(6,3)}=\partial_{X}^{-1}\left(\left(U_{X}^{(0)}\right)^{2} q^{(1)}\right)
\end{aligned}
$$




\section{Solution procedure}

To solve Eq. (27) for $U(T, X)$, through $O\left(\mu^{2}\right)$, one uses Eq. (28), with $U^{(1)}$ and $U^{(2)}$ given by Eqs. (33)-(35). The Normal Form, Eq. (29), is exploited in order to express derivatives of $U^{(0)}$ with respect to $T$ as differential polynomials in $U^{(0)}$ (polynomials in $U^{(0)}$ and its derivatives with respect to $X$ ). One then solves for the coefficients appearing in Eqs. (33) and (34) order-by-order.

\subsection{Solution when $U^{(0)}$ is a single soliton}

Owing to the simple structure of $U^{(0)}$, only a few of the coefficients in Eqs. (33) and (34) can be determined when $U^{(0)}$ is a single soliton, given by Eqs. (31) and (32). They are given below.

$O(\varepsilon)$

$$
\begin{gathered}
c_{1}=\left(-\frac{11}{12 \alpha}+\frac{3}{8} \rho \alpha\right)+c_{2}-c_{3}+4 c_{5}+\frac{1}{3} c_{6} \\
c_{4}=\left(-\frac{1}{2 \alpha}+\frac{3}{4} \rho \alpha\right)+c_{2}-c_{3}+8 c_{5}+\frac{5}{3} c_{6}+\frac{1}{2} c_{7}
\end{gathered}
$$

$\underline{O\left(\varepsilon^{2}\right)}$

$$
\begin{aligned}
d_{1}= & \frac{529}{1440 \alpha^{2}}-\frac{1}{3 \alpha}\left(3 c_{5}+c_{6}\right)+\rho\left(-\frac{19}{48}+\frac{27}{128} \alpha^{2}+\frac{1}{2} \alpha\left(3 c_{5}+c_{6}\right)\right) \\
& +d_{2}-d_{3}+d_{4}+\frac{1}{3} d_{5}-d_{6}-\frac{1}{3} d_{7}+\frac{2}{15} d_{17}+\frac{1}{15} d_{18}+\frac{1}{15} d_{19}+d_{21}+6 d_{23} \\
d_{9}= & \frac{3}{2 \alpha^{2}}+\rho\left(-\frac{13}{8}+\frac{9}{16} \alpha^{2}\right)+4 d_{2}-4 d_{3}+4 d_{4}+\frac{4}{3} d_{5}-4 d_{6}-\frac{4}{3} d_{7}-\frac{1}{2} d_{8} \\
& +d_{13}-\frac{1}{3} d_{14}-\frac{1}{3} d_{15}-\frac{1}{3} d_{16}-\frac{8}{15} d_{17}-\frac{4}{15} d_{18}-\frac{4}{15} d_{19}+\frac{4}{3} d_{21}-\frac{8}{9} d_{22}+16 d_{23} \\
d_{1}= & \frac{41}{240 \alpha^{2}}-\frac{5}{\alpha}\left(3 c_{5}+c_{6}\right)+\rho\left(-\frac{71}{32}+\frac{9}{4} \alpha^{2}+\frac{15}{2}\left(3 c_{5}+c_{6}\right) \alpha\right) \\
& +3 d_{2}-6 d_{3}+7 d_{4}+\frac{10}{3} d_{5}-7 d_{6}-\frac{10}{3} d_{7}+\frac{1}{2} d_{8}+d_{11}-d_{12}-d_{13}+\frac{1}{3} d_{14} \\
& +\frac{5}{6} d_{15}+\frac{5}{6} d_{16}+\frac{13}{5} d_{17}+\frac{9}{5} d_{18}+\frac{9}{5} d_{19}+\frac{1}{2} d_{20}+\frac{41}{3} d_{21}+\frac{11}{9} d_{22}+62 d_{23} \\
a_{2}= & -\frac{1}{144 \alpha^{2}}+\rho \frac{1}{32}-\frac{1}{3 \alpha}\left(3 c_{5}+c_{6}\right)+\frac{4}{5} d_{17}+\frac{2}{5} d_{18}+\frac{2}{5} d_{19}+2 d_{21}+\frac{2}{3} d_{22}+6 d_{23}
\end{aligned}
$$

The fictitious parameter, $\rho(=1)$, singles out the relativistic effects and $\alpha$-the effect of the average electron kinetic energy. Finally, for a general distribution function, $\psi$, in Eq. (3), the solutions for the coefficients in Eqs. (36) and (37) are modified. For example, $a_{1}$ and $a_{2}$ are then given by:

$$
\begin{aligned}
a_{1}=\frac{1}{4 \alpha} & \left(\left(3 \psi^{\prime}(0)^{2}-\psi^{\prime \prime}(0)\right) /\left(2 \psi^{\prime}(0)^{3 / 2}\right)\right)+2\left(3 c_{5}+c_{6}\right) . \\
a_{2}= & -\frac{1}{144 \alpha^{2}} \frac{36 \psi^{\prime}(0)^{2} \psi^{\prime \prime}(0)-27 \psi^{\prime}(0)^{4}-4 \psi^{\prime \prime}(0)^{2}-3 \psi^{\prime}(0)}{2 \psi^{\prime}(0)^{3}}+\rho \frac{1}{32} \\
& -\frac{1}{3 \alpha}\left(3 c_{5}+c_{6}\right) \frac{3 \psi^{\prime \prime}(0)-\psi^{\prime}(0)^{2}}{2 \psi^{\prime}(0)^{3 / 2}}+\frac{4}{5} d_{17}+\frac{2}{5} d_{18}+\frac{2}{5} d_{1 \%}+2 d_{21}+\frac{2}{3} d_{22}+6 d_{23}
\end{aligned}
$$

The computation reveals that when $U^{(0)}$ is a single-soliton, no obstacle to integrability emerges in $U^{(n)}, n \geq 1$. Hence, in Eq. (34),

$$
W(T, X)=0
$$


Owing to the simple functional form of the single-soliton solution, one may assign the undetermined coefficients in Eqs. (36) and (37) any values. All choices are equivalent. In particular, one may choose the undetermined coefficients to vanish. With this choice, Eqs. (36) and (37) yield the following expressions for $a_{1}$ and $a_{2}$ in the Normal Form, Eq. (29):

$$
a_{1}=\frac{1}{4 \alpha} \quad, \quad a_{2}=-\frac{1}{144 \alpha^{2}}+\rho \frac{1}{32} .
$$

The constraints imposed in the case of a general solution yield for $a_{1}$ and $a_{2}$ (see Section 6.2.), the same expressions as in Eq. (39) (although then not all the undetermined coefficients in Eqs. (36) and (37) vanish). Note that the second-order correction to the soliton velocity is affected by the relativistic effect (Again, $\rho=1$ ).

Finally, the expressions for the coefficients in Eqs. (36) and (37) when all undetermined coefficients are set to zero will be required in Section 8:

$$
\begin{aligned}
& c_{1}=\left(-\frac{11}{12 \alpha}+\frac{3}{8} \rho \alpha\right), \quad c_{4}=\left(-\frac{1}{2 \alpha}+\frac{3}{4} \rho \alpha\right) \\
& d_{1}=\frac{529}{1440 \alpha^{2}}+\rho\left(-\frac{19}{48}+\frac{27}{128} \alpha^{2}\right), d_{9}=\frac{3}{2 \alpha^{2}}+\rho\left(-\frac{13}{8}+\frac{9}{16} \alpha^{2}\right) . \\
& d_{10}=\frac{41}{240 \alpha^{2}}+\rho\left(-\frac{71}{32}+\frac{9}{4} \alpha^{2}\right)
\end{aligned}
$$

\subsection{Perturbation analysis for general $U^{(0)}$}

\subsubsection{Freedom in analysis}

There is still ample freedom in solving for the coefficients in Eqs. (33) and (34) even when $U^{(0)}$ is a multi-soliton solution. The choice made here is to adopt Eqs. (36) and (37), obtained in the single-soliton analysis. One now solves for all remaining, yet undetermined, coefficients.

The rationale behind this choice is that, away from the soliton interaction region, a multi-soliton solution breaks up into a sum of well-separated single solitons. Hence, barring exponentially small terms, the single-soliton results of Section 6.1 apply in the vicinity of each single soliton; Eq. (27) is obeyed asymptotically far from the interaction region for any values assigned to the undetermined coefficients in Eqs. (36) and (37). Consequently, the results one now obtains for the coefficients that have remained undetermined in the single-soliton analysis and for $W(T, X)$ represent the effect of soliton interactions, which are confined to a finite domain in the $x$-t plane.

\subsubsection{Resulting coefficients}

To solve for $U^{(1)}$ and $U^{(2)}$, one exploits the Normal Form, Eq. (29), repeatedly in order to express any derivative of $U^{(0)}$ with respect to $T$ as a differential polynomial in $U^{(0)}$ and its spatial derivatives. The results for the coefficients in Eqs. (33) and (34) are:

$$
\begin{aligned}
& c_{1}=\left(\frac{1}{4 \alpha}+\rho \frac{3}{8} \alpha\right), \quad c_{2}=\frac{7}{6 \alpha}, c_{4}=\left(\frac{2}{3 \alpha}+\rho \frac{3}{4} \alpha\right), \\
& d_{1}=-\frac{9221}{4320 \alpha^{2}}+\rho\left(\frac{7}{24}+\frac{171}{128} \alpha^{2}\right), d_{2}=-\frac{1967}{1080 \alpha^{2}}+\rho\left(\frac{5}{12}+\frac{9}{8} \alpha^{2}\right), d_{3}=\frac{49}{72 \alpha^{2}} \\
& d_{5}=\rho \frac{13}{16}, d_{9}=-\frac{2297}{270 \alpha^{2}}+\rho\left(\frac{121}{48}+\frac{81}{16} \alpha^{2}\right), d_{10}=-\frac{6751}{720 \alpha^{2}}+\rho\left(\frac{63}{32}+\frac{45}{8} \alpha^{2}\right) . \\
& d_{11}=\rho \frac{13}{8}, d_{13}=\rho \frac{67}{48}
\end{aligned}
$$

The remaining coefficients are found to vanish. The expressions for $a_{1}$ and $a_{2}$ in the Normal Form, Eq. (29), are given in Eq. (39). Note, again, that the relativistic effects (multiplied by $\rho=1$ ) and the effect of the average electron kinetic energy (through $\alpha$ ) are explicitly separated.

\section{Long-range interactions amongst solitons when $U^{(0)}$ is a multi-soliton solution}

When the zero-order solution, $U^{(0)}$, is a multi-soliton solution, interactions amongst solitons in $U^{(0)}$ are confined to the finite soliton collision region. Away from this domain, $U^{(0)}$ breaks up into a sum of well-separated single solitons. Hence, 
in any contribution of a monomial in $U^{(0)}$ or its spatial derivatives (e.g., $\left(U^{(0)}\right)^{2} U_{X}^{(0)}$ ) to the higher-order corrections, $U^{(n)}, n \geq 1$, in Eq. (28), soliton interactions are also confined to the soliton collision region.

However, both the first- and second-order corrections in Eq. (28) contain terms that involve the nonlocal entities of Eq. (35). Such entities induce long-range interactions among different solitons. The effect emerges already in $U^{(1)}$, through the term $c_{2} U_{X}^{(0)} q^{(1)}$ in Eq. (33), with $q^{(1)}$ defined in Eq. (35). Hence, it is discussed as an example. Figure 1 shows a two-soliton solution, the expression for which is given by:

$$
\begin{aligned}
& U^{(0)}(T, X)=2 \partial_{X}^{2} \log f(T, X) \\
& f(T, X)=1+g(T, X, 1)+g(T, X, 2)+\left(\frac{k_{2}-k_{1}}{k_{2}+k_{1}}\right)^{2} g(T, X, 2) g(T, X, 2) . \\
& g(X, T, n)=e^{2 \xi_{n}} \quad, \quad \xi_{n}=k_{n}\left(X+4 k_{n}^{2} T+\delta_{n}\right)
\end{aligned}
$$

Figure 2 shows a plot of the same two-soliton solution looked at from above. The numbers 1 and 2 on the soliton lines represent the soliton wave numbers, $k_{1}<k_{2}$. Focusing on large $T<0$, the term $U_{X}^{(0)} q^{(1)}$ vanishes asymptotically in the regions denoted by $i$, ii and $v$. In regions iii and $i v$ one has:

$$
U_{Y}^{(0)} q^{(1)} \cong\left\{\begin{array}{ll}
\text { iii }: & -8 k_{1}{ }^{4} \frac{\sinh \left[\xi_{1}\right]^{2}}{\cosh \left[\xi_{1}\right]^{4}} \\
\text { iv : } & -8 k_{1} k_{2}{ }^{3} \frac{\sinh \left[\xi_{2}\right]}{\cosh \left[\xi_{2}\right]^{3}}
\end{array} \quad, \quad(\xi=X+V T+\delta)\right.
$$

The nonlocal term generates long-range interactions amongst solitons; the contribution around soliton no. 2 is affected by $k_{1}$. Note that the fictitious coefficient, $\rho$, is absent in the non-local term in $U^{(1)}$. Hence, this non-local effect is the same in the non-relativistic and the relativistic limits. Similar results are found at large $T>0$, with the roles of $k_{1}<k_{2}$ interchanged. Nonlocal effects of a similar nature are generated in $U^{(2)}$ through the terms $d_{2} U_{X X X}^{(0)} q^{(1)}+d_{3} U_{X X}^{(0)}\left(q^{(1)}\right)^{2}$.

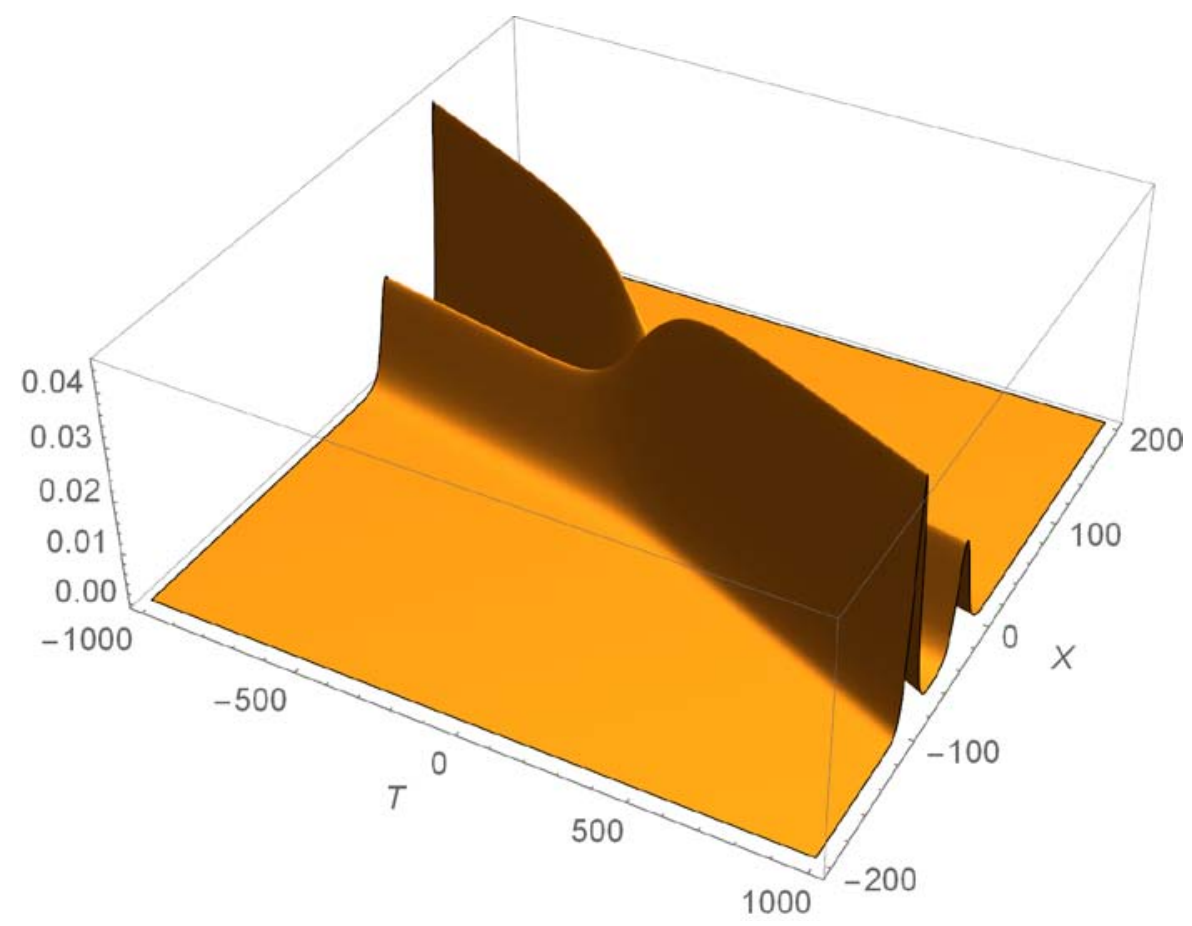

Figure 1. Two-soliton solution (Eq. (43)). $k_{1}=0.1, k_{2}=0.15, \delta_{1}=\delta_{2}=0$. 


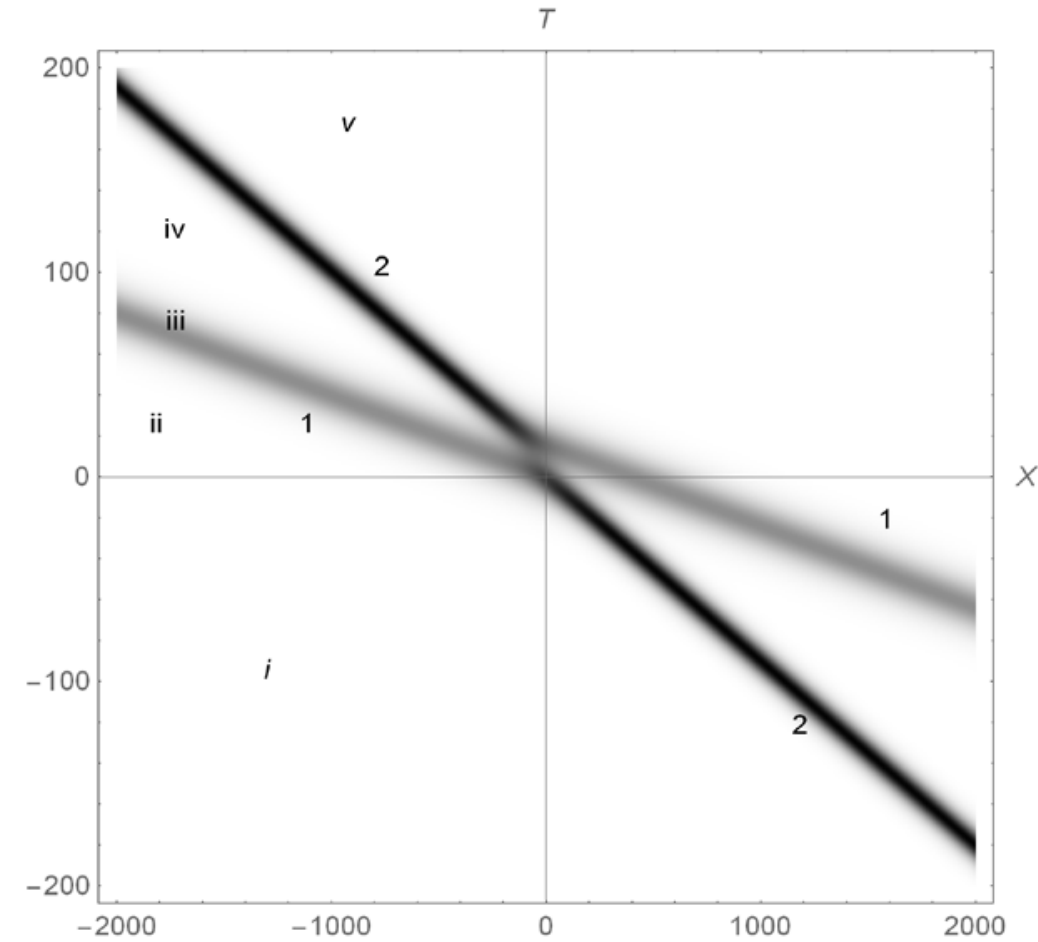

Figure 2. Density plot of two-soliton solution of Figure 1 with regions of interest in the $x$-t plane indicated.

\section{Effect of soliton interaction region when $U^{(0)}$ is a multi-soliton solution}

When $U^{(0)}$ is a multi-soliton solution, soliton interactions, which occur in the finite domain of soliton interactions in the $x-t$ plane, affect the correction terms, $U^{(n)}, n \geq 1$. Some effects are mere addition of localized humps to $U^{(n)}$. However, from second order onwards, a new phenomenon emerges. The soliton interaction region generates dispersive waves in $U^{(n)}$.

\subsection{Localized hump in first-order correction}

Exploiting the coefficients given in Eq. (41), one re-writes $U^{(1)}$ of Eq. (33) in the following form:

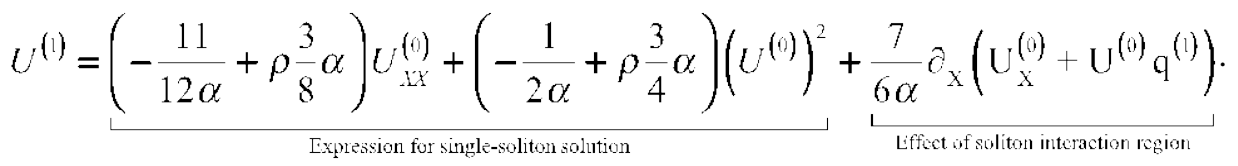

The first term has the form of the contribution of a single-soliton solution to $U^{(1)}$ (see Eq. (33)), with all the undetermined coefficients in Eq. (36) set to zero (a valid choice, see Eq. (41)). The second term in Eq. (45) vanishes identically when $U^{(0)}$ is a single-soliton solution. As a result, when $U^{(0)}$ is a multi-soliton solution, this second term adds to $U^{(1)}$ a hump that is localized around the soliton interaction region. The reason is that, away from the soliton interaction region, a multi-soliton $U^{(0)}$ splits into a sum of well-separated single solitons. Hence, along each soliton, the second term in Eq. (45) term vanishes asymptotically. As the second term in Eq. (45) is not multiplied by $\rho(=1)$, the contribution of the localized hump to $U^{(1)}$ exists both in the non-relativistic and in the weak relativistic limits. by

For a general distribution, $\psi$ in Eq. (3), the effect of second term in Eq. (45) remains the same. It is merely multiplied

$$
\left(13 \psi^{\prime}(0)^{2}+\psi^{\prime \prime}(0)\right) /\left(14 \psi^{\prime}(0)^{3 / 2}\right)
$$

\subsection{Dispersive waves in second-order corrections}

Effects of a similar nature to that discussed in Section 8.1 emerge also in the second-order analysis. $U^{(2)}$ contains closed form contributions to (differential polynomials in $U^{(0)}$ ), which are humps that are localized around the soliton interaction region. As they are of a similar nature to what has been just discussed in the case of the first-order correction, 
they are not discussed here.

A new type of soliton interactions emerges in second order. It has no first-order counterpart. The equation for $U^{(2)}$ contains an obstacle to asymptotic integrability, a driving term, the effect of which cannot be accounted for by the most general differential polynomial possible employed in Eq. (34). It is responsible for the generation of $W(T, X)$ in Eq. (34). The solution procedure of Section 6.2, ensures that the effect of the obstacle is confined to the finite soliton interaction region.

The resulting equation for $W(T, X)$ is:

$$
W_{T}=6\left(U^{(0)} W\right)_{X}+W_{X X X}+\rho\left(\partial_{X} R_{0}+\alpha^{2} R_{1}\right)+\frac{1}{\alpha^{2}} R_{2} .
$$

In Eq. (46),

$$
\begin{aligned}
R_{0}= & -\frac{15}{16} U^{(0)}\left(\left(U^{(0)}\right)^{3}-\left(U_{X}^{(0)}\right)^{2}+U^{(0)} U_{X X}^{(0)}\right), \\
R_{1}= & \partial_{X} R_{10}+q^{(1)} \partial_{X X} R_{11}, \\
R_{10}= & \left\{\begin{array}{l}
\frac{27}{16}\left(U^{(0)}\right)^{4}+\frac{135}{4} U^{(0)}\left(U_{X}^{(0)}\right)^{2}-\frac{27}{8}\left(U^{(0)}\right)^{2} U_{X X}^{(0)} \\
+\frac{27}{4}\left(U_{X X}^{(0)}\right)^{2}+\frac{135}{8} U_{X}^{(0)} U_{X X X}^{(0)}-\frac{27}{8} U^{(0)} U_{X X X X}^{(0)}
\end{array}\right\}, \\
R_{11}= & \frac{81}{8}\left(U_{X}^{(0)}\right)^{2} \\
R_{2}= & \partial_{X} R_{20}+q^{(1)} \partial_{X} R_{21}, \\
R_{20}= & -\frac{37}{72}\left(U^{(0)}\right)^{4}-\frac{19453}{360} U^{(0)}\left(U_{X}^{(0)}\right)^{2}+\frac{3097}{360}\left(U^{(0)}\right)^{2} U_{X X}^{(0)} \\
& -\frac{577}{48}\left(U_{X X}^{(0)}\right)^{2}-\frac{5087}{180} U_{X}^{(0)} U_{X X X}^{(0)}+\frac{1087}{360} U^{(0)} U_{X X X X}^{(0)}-\frac{7}{72} U_{X X X X X X}^{(0)}, \\
R_{21}= & -\frac{7}{2}\left(U^{(0)}\right)^{2} U_{X}^{(0)}-\frac{931}{30} U_{X}^{(0)} U_{X X}^{(0)}-\frac{7}{6} U^{(0)} U_{X X X}^{(0)}-\frac{7}{72} U_{X X X X X}^{(0)}
\end{aligned}
$$

Substituting the single-soliton solution, Eq. (31) one finds that $R_{0}, R_{1}$ and $R_{2}$ all vanish identically. Consequently, when $U^{(0)}$ is a multi-soliton solution, each of these driving terms is localized around the soliton interaction region, and vanishes exponentially fast along each soliton away from that region. In addition, one sees how the roles played by the different terms in Eq. (46) change as $\alpha$ varies (i.e., when the average electron kinetic energy is varied). Finally, only $R_{2}$ exists both in the nonrelativistic well as in the weak relativistic limits. All other driving terms are multiplied by $\rho(=1)$, signifying that they are generated by relativistic effects.

As an example, consider the effect of $R_{0}$. A plot of $R_{0}$ is shown in Figure 3, for the two-soliton solution of Figure 1. Denoting the contribution of $R_{0}$ to $W(T, X)$ by $W_{0}$, the equation for the latter is

$$
W_{0, T}=6\left(U^{(0)} W_{0}\right)_{X}+W_{0, X X X}+\partial_{X} R_{0} .
$$

Defining

$$
W_{0}=\partial_{X} \omega,
$$

The equation for $\omega$ is:

$$
\omega_{T}=6 U^{(0)} \omega_{X}+\omega_{X X X}+R_{0} .
$$

The numerical solution for $\omega$ is shown in Figure 4 with $U^{(0)}$-the two-soliton solution of Figure 1. Figure 5 displays the $X$-dependence of $\omega$ (full line) at large $T>0(T=4,000$, i.e., far from the soliton interaction region). The computation reveals that $\omega$ contains the profiles of the two original solitons of Eq. (43), each modified by a numerical coefficient, and a trailing dispersive wave. (A simple numerical exercise shows that any driving term in Eq. (54) that is a hump, which is sufficiently localized around the soliton collision region, e.g., a Gaussian that decays away from that region, will generate 
such an effect). The dashed line represents the sum of the profiles of the two single solitons, each multiplied by the numerical factor found from the computation of $\omega\left(0.00473641\right.$ for the $k_{2}$ soliton, and -0.00527162 for the $k_{1}$ soliton). With these multiplicative factors, the soliton profiles in $\omega$ and the actual soliton profiles coincide. Figure 5 shows how $\omega$ differs from the sum of the profiles of the two solitons owing to the emergence of the dispersive wave. A detailed plot of the dispersive wave is shown in Figure 6. The possibility of the generation of dispersive waves was pointed out in numerical studies of the perturbed KdV equation years ago [24, 35, 36].

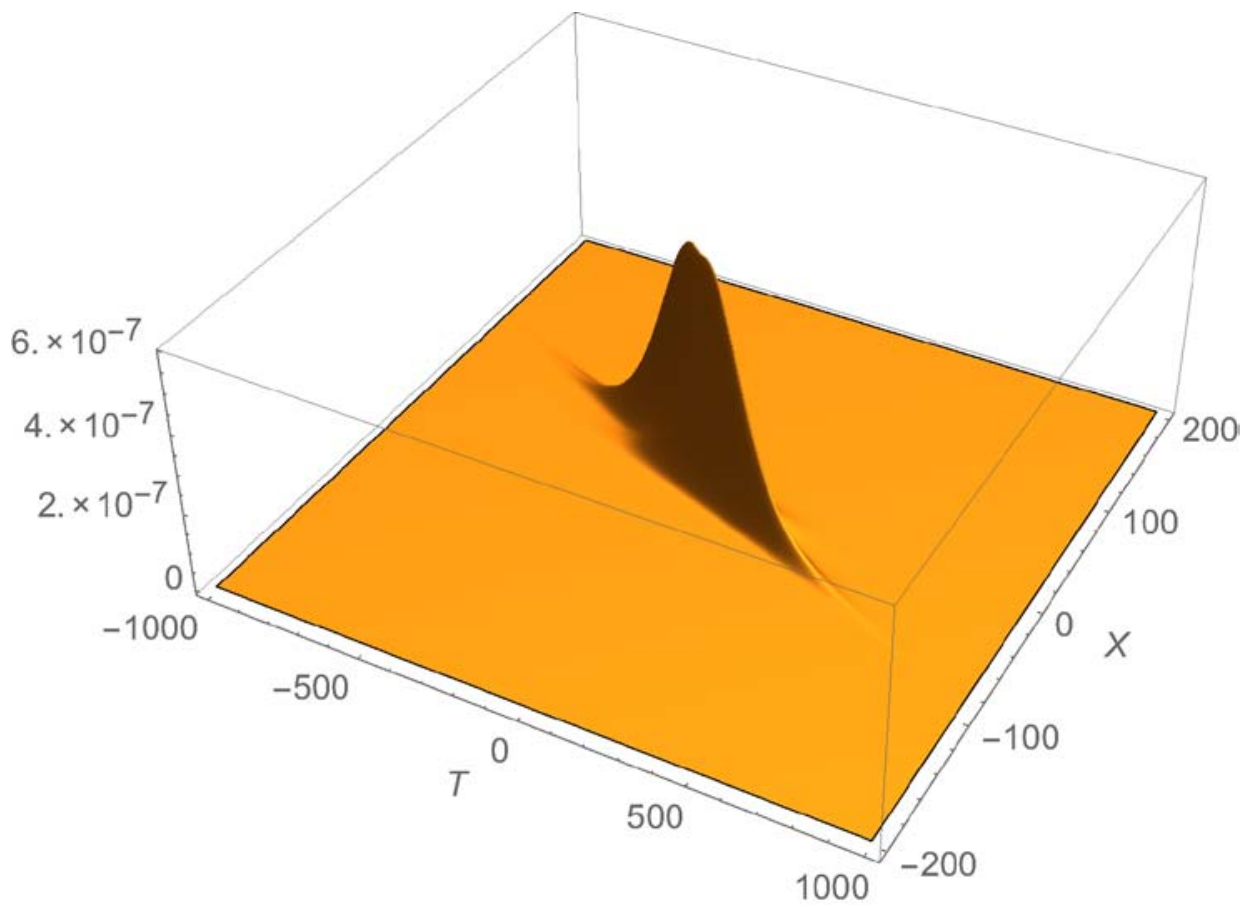

Figure 3. $\boldsymbol{R}_{\mathbf{0}}$ (Eq. (47)) for two-soliton solution of Figure 1.

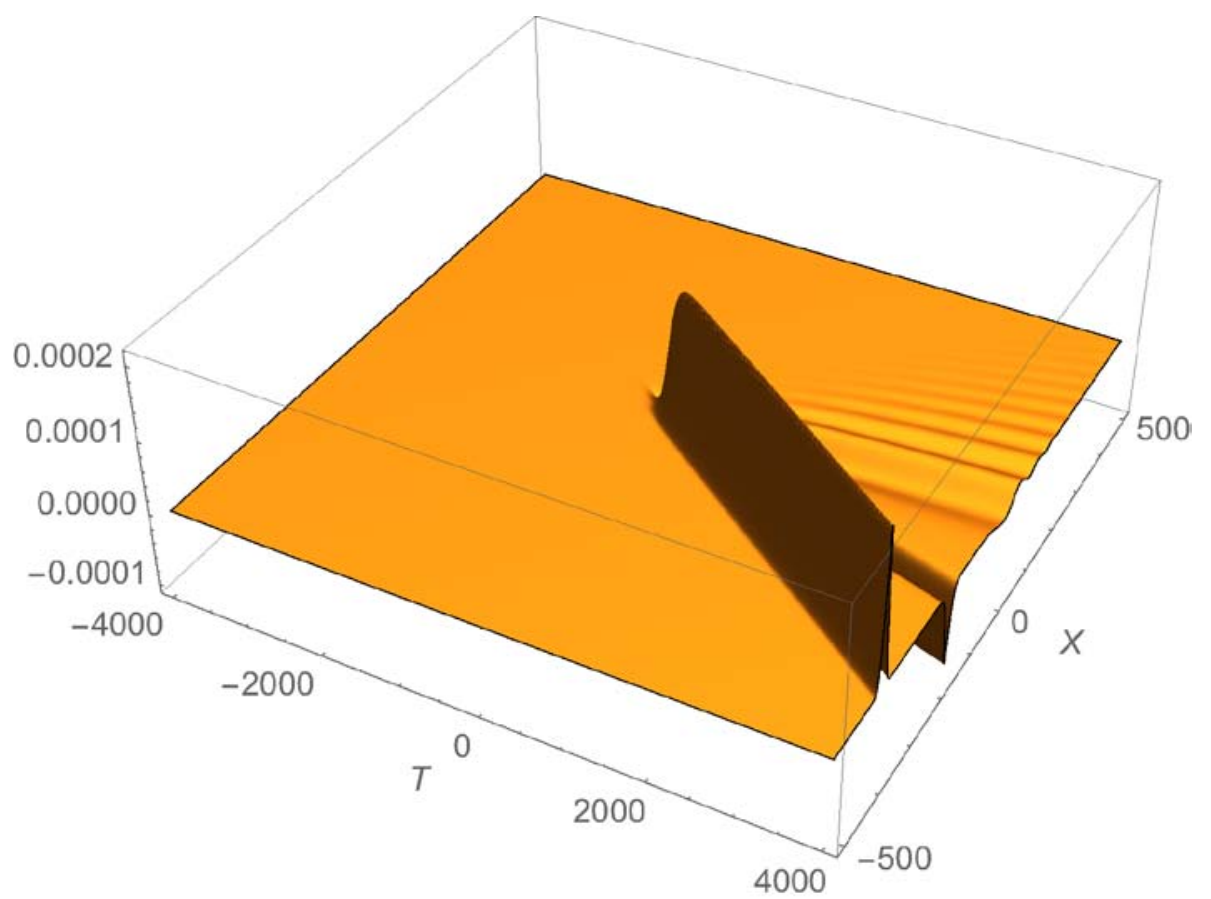

Figure 4. Numerical solution of Eq. (54) for $\omega$ of Eq. (53) in case of two-soliton solution of Figure 1. 


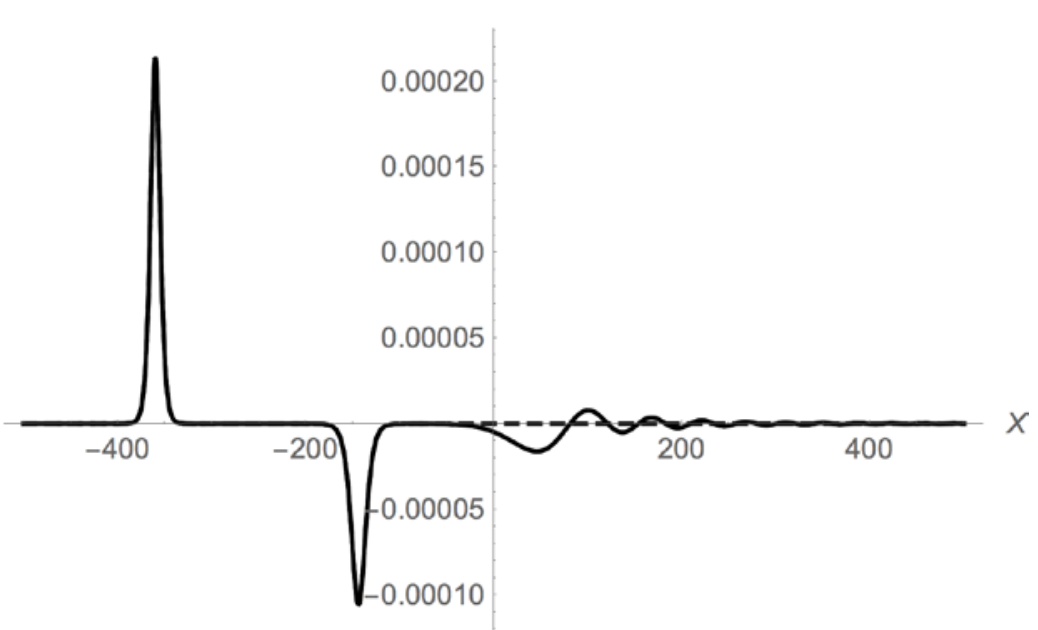

Figure 5. $X$ dependence at $T=4000$. Full line: profile of $\omega$ of Figure 4. Dashed line: sum of two soliton profiles.

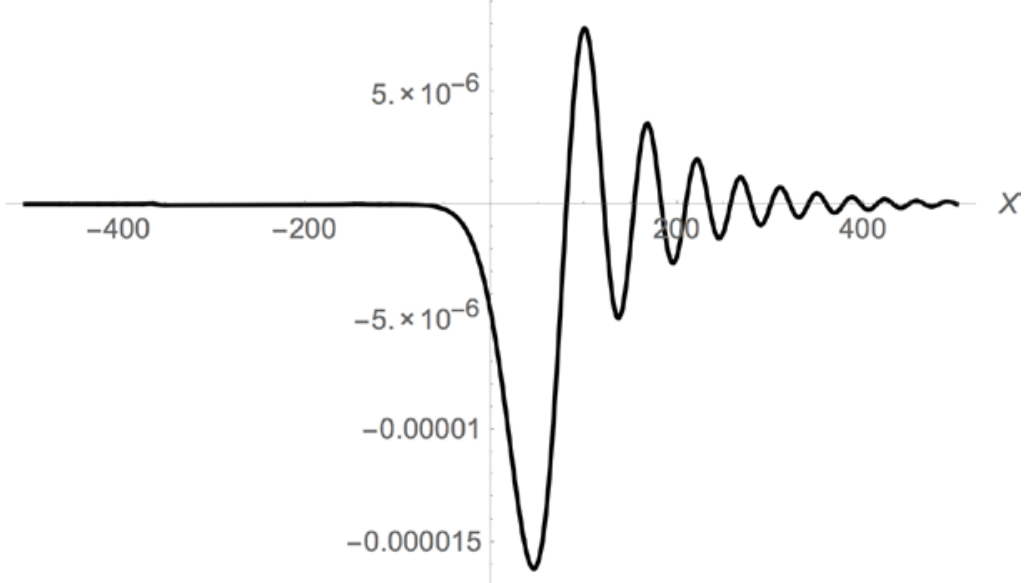

Figure 6. Profile of dispersive wave part in $\omega$ (Eqs. (53) and (54)) at $T=4000$.

Finally, two comments are in order here. First, each of the driving terms in Eq. (46) generates in $W$ contributions of a similar nature. Second, for a general distribution, $\psi$, in Eq. (3), the effects remain, with modified coefficients. For example, for a general $\psi, R_{0}$ of Eq. (47) is multiplied by

$$
2 \psi^{\prime}(0)^{2} /\left(3 \psi^{\prime}(0)^{2}-\psi^{\prime \prime}(0)\right)
$$

\section{Effect of average electron kinetic energy}

The effect of the average electron kinetic energy, $\left(k_{B} \cdot T_{e}\right)$, enters through $\alpha$ of Eq. (5). The analysis presented here allows for the study of the effect when $\alpha \ll 1$, or $\alpha=O(1)$.

When $\alpha \ll 1$, in all corrections to the solution, as well as to the soliton velocity, the contributions with negative powers of $\alpha$ dominate; the remaining contributions are negligible. For example, using Eq. (39), to a very good approximation, the updated soliton velocity of Eq. (32) becomes:

$$
V=4 q^{2}\left(1+\left(\mu q^{2} / \alpha\right)-\frac{1}{9}\left(\mu q^{2} / \alpha\right)^{2}+\ldots\right)
$$

A similar situation exists in the higher order corrections to the solution. For example, Eq. (45) for $U^{(1)}$ is well approximated by

$$
U^{(1)} \simeq \frac{1}{2 \alpha}\left\{\left(-\frac{11}{6}\right) U_{x x}^{(0)}-\left(U^{(0)}\right)^{2}+\frac{7}{3} \partial_{x}\left(\mathrm{U}_{x}^{(0)}+\mathrm{U}^{(0)} \mathrm{q}^{(1)}\right)\right\}
$$


$U^{(0)}$ is proportional to $q^{2}$. Hence, all terms in Eq. (56) are $O\left(q^{4}\right)$. Thus, whereas $\mu$ represents the smallness of $(v / c)$, the effective expansion parameter in both Eqs. (55) and (56) is:

$$
\tilde{\mu}=\left(\mu q^{2} / \alpha\right)
$$

$\tilde{\mu}$ varies as $\alpha$ is increased. When $\alpha=O(1)$, it becomes $\mu q^{2}$. The approximation of Eq. (55) is now replaced by Eqs. (32) and (39) and that of Eq. (56) - by Eqs. (32), (39) and (45). For the perturbation expansion to be of significance $\tilde{\mu}$ must be sufficiently small.

\section{Concluding comments}

The physically relevant scaling of Eq. (8) reflects the smallness of the wave velocity, $v$, of Eqs. (1-3) relative to the speed of light, $c$. It allows for the separate study of non-relativistic and relativistic effects, as well as of the effect of the average electron kinetic energy on the characteristics of the solution. The second-order analysis reveals the emergence of dispersive waves that are not soliton-like. The qualitative results do not depend on the details of the thermodynamic distribution of the electrons.

\section{References}

[1] K. K. Ghosh and D. Ray. (1991). Phys. Fluids, B 3, 300-303.

[2] P. Chatterjee and R. Roychoudhury. (1994). Phys. Plasmas, 1, 2148-2153.

[3] R. Gogoi, N. Devi, and G. C. Das. (2008). Ind. J. Pure \& Appl. Phys., 46, 621-628.

[4] R. S. Ibrahim and O. H. El-Kalaawy. (2008). Adv. Studies. Theor. Phys., 2, 919-928.

[5] A. Shah and R. Saeed. (2009). Phys. Lett., A 373, 4164-4168.

[6] H. R. Pazkad and K. Javidan. (2011). Astrophys. Space Sci., 331, 175-180.

[7] B. Sahu. (2011). Pranama J. Phys., 76, 933-944.

[8] A. Rahman, I. Kourakis, and A. Qamar. (2015). IEEE Trans. Plasma Sci., 43, 974-984.

[9] S. Chandra, S. Das, A. Chandra, B. Ghosh, and A. Jash. (2015). Int'l J. Nucl. Quant. Eng., 9, 305-311.

[10] M. R. Hossen, M. A. Hossen, S. Sultana, and A. A. Mamun. (2015). Astrophys. Space Sci., 357(34), 1-8.

[11] M. G. Hafez, M. R. Talukder, and M. Hossain Ali. (2016). Waves in Random \& Complex Media, 26, 68-80.

[12] V. Yu. Belashov. (2017). Geomagnetism \& Aeronomy, 57, 307-311.

[13] I. S. Elkamash, I. Kourakis, and F. Haas. (2017). Phys. Rev., E96, 043206.

[14] B. Hosen, M. G. Shah, M. R. Hossen, and A. A. Mamun. (2018). Plasma Phys. Rep., 44, 976-985.

[15] M. Kr. Deka, A. N. Dev, A. P. Misra, and N. C. Adhikary. (2018). Phys. Plasmas, 25, 012102.

[16] S. K. El-Labany, M. S. Abdel Krim, S. A. El-Warraki, and W. F. El-Taibany. (2003). Chinese Phys., 12, 759-764.

[17] C. S. Panguetna, C. B. Tabi, and T. C. Kofané. (2019). J. Theor. \& Appl. Phys., 13, 237-249.

[18] G. C. Das and S. N. Paul. (1985). Phys. Fluids, 28, 823-825.

[19] G. C. Das, B. Karmakar, and S. N. Paul. (1988). IEEE Trans. Plasma Sci., 16, $22-26$.

[20] E. F. EL-Shamy, E. K. El-Shewy, N. F. Abdo, M. Ould Abdellahi, and O. Al-Hagan. (2019). Contrib. Plasma Phys., 59, 304-313.

[21] Fokas, A. S. and Liu, Q. M. (1996). Phys. Rev. Lett., 77, 2347-2351.

[22] Kodama, Y. (1985). Phys. Lett., A 112, 193-196.

[23] Kodama, Y. (1985). Physica, D 16, 14-26.

[24] Marchant, T. R. and Smyth, N. F. (1996). IMA J. Appl. Math., 56, 157-176.

[25] Kodama, Y. (1987). Normal Form and Solitons, pp. 319-340 in Topics in Soliton Theory and Exactly Solvable Nonlinear Equation, ed. by M.J. Ablowitz et al. (World Scientific, Singapore, 1987).

[26] Hiraoka, Y. and Kodama, Y. (2002). Normal Form and Solitons, Lecture notes, Euro Summer School 2001, The Isaac Newton Institute, Cambridge, August 15-25 (2002). 
[27] Marchant, T. R. (2002). Stud. Appl. Math., 109, 1-17.

[28] Mikhailov, A. V. (1991). Talks at NEEDS-91 Conf., Gallipoli, Italy (1991); NATO Adv. Res. Workshop; Singular limits of Dispersive waves, Lyon, France (1991) (unpublished).

[29] Kodama, Y. and Mikhailov, A. V. (1997). Obstacles to Asymptotic Integrability, pp. 173-204 in Algebraic Aspects of Integrable Systems, ed. by A. S. Fokas \& I. M. Gelfand (Birkhäuser, Boston, 1997).

[30] Fokas, T. \& Luo, L. (1996). Contemp. Math., 200, 85-98.

[31] Kraenkel, R. A., Pereira, J. G., and de Rey Neto, E. C. (1998). Phys. Rev., E58, 2526-2530.

[32] Marchant, T. R. (2002). Phys. Rev., E66, 046623, 1-8.

[33] Veksler, A. and Zarmi, Y. (2004). WSEAS Trans. Math., 3, 560-565.

[34] Y. Zarmi. (2008). Physica, D 237, 2987-3007.

[35] R. M. Mirie and C. H. Su. (1982). J. Fluid Mech., 115, 475-492.

[36] Q. Zou and C. H. Su. (1986). Phys. Fluids, 29, 2113-2123. 